\title{
The SECOM Platform: an Integrated CLEM Solution
}

\author{
S.V. den Hoedt ${ }^{1}$, A.P.J. Effting ${ }^{1}$, M.T. Haring ${ }^{1}$ \\ ${ }^{1}$ DELMIC BV, Delft, The Netherlands
}

The SECOM platform enables accurate and straightforward correlative light and electron microscopy (CLEM) by integrating a fluorescence microscope in a scanning electron microscope (SEM) ${ }^{1}$. The SECOM platform can be fitted on a SEM by replacing the door to the vacuum chamber of the SEM. This replacement supports a motorized stage, the objective and the light path for the optical microscope. The integration of the two imaging modalities eliminates the need to switch between the optical and electron microscope and allows for faster imaging and a more logical workflow. More importantly, the SECOM platform makes it possible to correlate two different types of information on the exact same cell, tissue or structure of interest ${ }^{2}$.

The SECOM platform can easily be integrated in the existing workflow and offers all features of a high-end optical microscope. Firstly, the sample stage of the SECOM platform is large, 18x18mm, meaning a wide range of samples can be accomodated. Secondly, vacuum compatible immersion oil developed by DELMIC can be used to obtain the same numerical apertures (1.4) known from conventional fluorescence microscopy. Lastly, correlation between electron microscopy and optical microscopy data is unrivalled and entirely automatic with the SECOM platform. The electron and light beam axes are aligned to within $0.2 \mu \mathrm{m}$ and, through proprietary methodology, an overlay accuracy of $50 \mathrm{~nm}$ or better can be achieved ${ }^{2}$.

Applications of the SECOM are primarily found in the life sciences. The SECOM platform is an excellent tool for research on tissue biology ${ }^{2}$ and thin sections. High quality fluorescence imaging allows for precise localization of different fluorescent labels, while the SEM delivers detailed structural information that surpasses traditional optical results, see figure 1 . In this talk, we will explain more about the technology of the SECOM platform and the possible application areas.

\section{References:}

[1] Zonnevylle, A.C. et al., Integration of a high-NA light microscope in a scanning electron microscope, Journal of Microscopy, 252, 58-70 (2013).

[2] Liv, N. et al., Simultaneous Correlative Scanning Electron and High-NA Fluorescence Microscopy, Plos One, 8(2), e55707 (2013). 


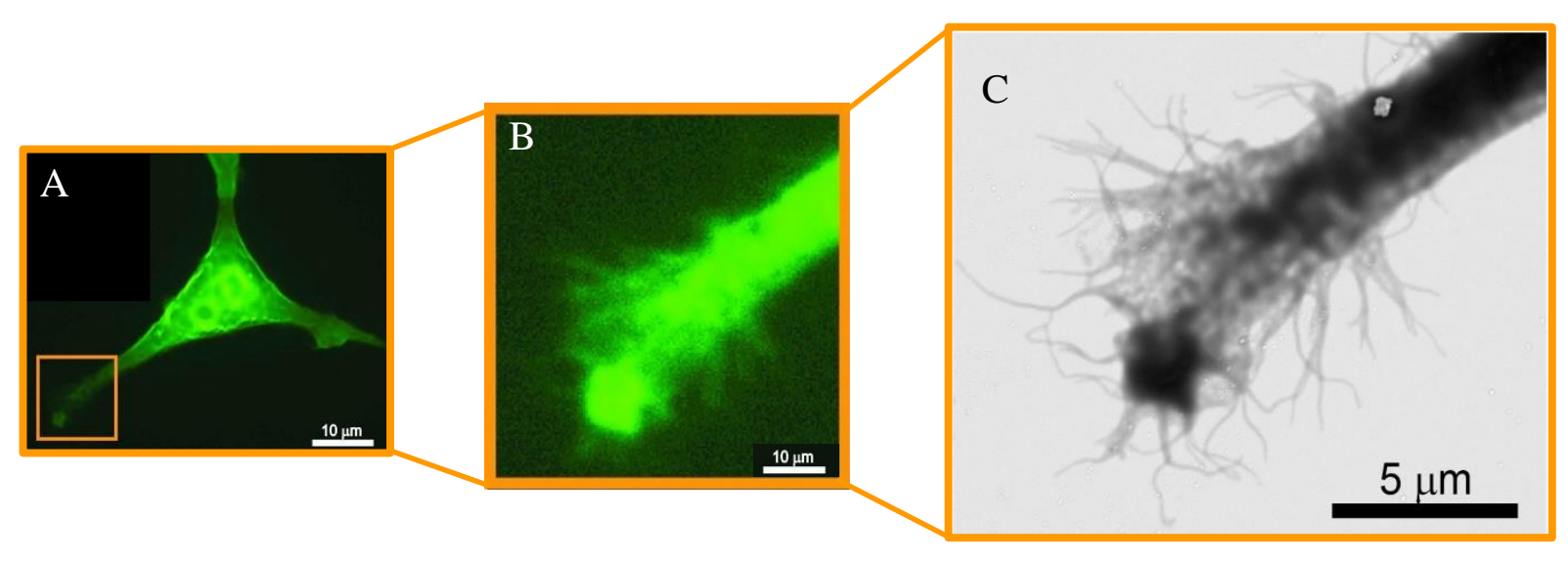

Figure 1. Simultaneous CLEM of whole uncoated cells. A,B) Fluorescence image of adenocarcinoma cells, actin is labeled with Alexa488, scale bar is $10 \mu \mathrm{m}$ C) SEM image of boxed area, scale bar is $5 \mu \mathrm{m}$. Image courtesy of N. Liv \& J.P. Hoogenboom 